\title{
Hypertonic Saline: A Review of the Literature and Evaluation of its Role in Resuscitation of Traumatic Hypovolaemic Shock
}

\author{
Maj K J Heath \\ FRCA, RAMC
}

Cambridge Military Hospital. Aldershot, Hants GU11 $2 A N$

SUMMARY: Hypertonic saline solutions have properties that may be advantageous for resuscitation. In both animal and human studies rapid improvements in cardiovascular status occur when hypertonic saline is administered to hypovolaemic subjects and the possible side effects of hypertonic solutions have not been shown to be of clinical importance. Hypertonic saline certainly has a potential role in resuscitation of hypovolaemic casulaties in the modern military setting.

\section{Introduction}

Aggressive intravenous fluid therapy remains the mainstay of treatment for hypovolaemic shock in both civilian and military practice. Intravenous fluids were used in the two world wars for resuscitation of hypovolaemic casualties. During the Falklands campaign (1), patients treated in the main casualty reception area, the SS Canberra, received a standard regime of 1 litre of compound sodium lactate followed by $500 \mathrm{mls}$ of polygeline. This regime was repeated if required then followed by cross matched blood. Of over 500 casualties treated, only 1 died of irreversible hypovolaemic shock. The nature of intravenous fluids used in resuscitation is controversial, but it is important that they contain sodium. The standard resuscitation fluids are crystalloid solutions isotonic with plasma. The Field Surgical Pocket Book (2) recommends 2-3 litres of crystalloid as initial resuscitation. A modern field ambulance carries both compound sodium lactate and colloids such as 'Haemacel' for resuscitation of casualties.

In the American urban setting pre-hospital fluid resuscitation has been challenged. Less than $1000 \mathrm{mls}$ were given during transport of casualties to hospital, suggesting that time spent siting intravenous cannulae is not worth the extra delay (3). It is the rapid transport of casualties to hospital rather than the administration of isotonic fluids, that are often insufficient to make up for blood loss, that is important. Pre-hospital fluid administration has been shown to have no impact upon survival following trauma especially if transportation times are under 30 minutes $(4,5)$.

There are more basic problems associated with fluid resuscitation in the military setting. 1 litre of fluid weighs 1 kilogram and for every 1 litre blood loss 3 litres of crystalloid are required to replace it thus creating large, heavy loads to accompany any resuscitation service, and adding even more weight to the already over-burdened modern soldier. Evacuation from a battlefield takes longer and is less straightforward than in a civilian setting and fluid resuscitation is indicated despite the fact that modern forms of transport, particularly helicopter, should considerably shorten evacuation times.
When considering the problem of resuscitation in military and also civilian practise, any agent that can be given rapidly, in small volumes and with equal or improved efficacy, is worth investigating.

Hypertonic saline solutions have been used clinically for nearly thirty years, although they have only more recently been evaluated in hypovolaemic shock. A review in 1985 (6) highlighted the potential advantages of hypertonic resuscitation fluid in the military setting, and theres has recently been a good deal of work using hypertoni $\overline{0}$ saline and hypertonic saline with dextran solutions i⿱亠巾禸 resuscitation of hypovolaemic traumatised patients. The्요 aim of this article is to review the current literature on the use of hypertonic saline for resuscitation in hypovolaemio shock and evaluate its potential role in the military set ting.

\section{Animal Studies with Hypertonic Saline}

Hypertonic saline has been used for the treatment of thermal injury for over 20 years and a $5 \%$ solution was described for treating Beurger's disease in 1926 (7).

In 1963 (8) hypertonic sodium solutions were shown to be effective in preventing the tissue damage caused by hypovolaemic shock in dogs, whereas isotonic solutions were not. In 1967 (9) it was demonstrated that small volumes of $7.5 \%$ and $5.2 \%$ sodium chloride, when compared with equal volumes of isotonic saline, produced improved cardiac output and mesenteric perfusion. More recently there is a good deal of animal work proving the efficacy of small volumes of hypertonic saline in producing a rapid improvement in arterial blood pressure and cardiac output in hypovolaemic animal models.

For example, dogs were bled to a level that would have resulted in $100 \%$ fatal shock (10). They were then resuscitated with either hypertonic saline or isotonic saline in a volume equal to $10 \%$ of the shed blood volume. In the hypertonic saline group arterial blood pressure and cardiac output were restored rapidly to normal levels and long term survival was improved. This was not the case in the group treated with isotonic saline. In another study (11) sheep were haemorrhaged 1.3-1.8 1 and resuscitated 
with $10 \%$ of this volume as hypertonic saline. They had a similar improved haemodynamic response, although in this study the effects were only transient. Similarly other studies (12) have shown marked but only transient improvements in cardiac output using small volumes of hypertonic saline for resuscitation.

The original studies therefore suggested that small volumes of hypertonic saline produced a better response in hypovolaemic animals than conventional fluids, although in these studies the comparison was between equal volumes of the different fluids.

In order to compare small volume resuscitation with conventional volumes, $4 \mathrm{mls} / \mathrm{kg}$ of $7.5 \%$ saline was given as a bolus over 5 minutes to conscious hypovolaemic swine (13). The response was compared to animals receiving a slow infusion of Ringer's lactate equivalent to $300 \%$ the shed blood volume over 1 hour. The group receiving hypertonic saline had a significant improvement of cardiac output initially and this effect was sustained for 2 hours.

The Ringer's lactate group on the other hand showed a gradual improvement in cardiac output to levels equal or better than the hypertonic saline group by the end of the 2 hours. Thus whilst large volumes of conventional fluids are effective for resuscitation, they take time to infuse. The rate of infusion through a 14 gauge cannula (not pressurised) is $250 \mathrm{mls} / \mathrm{min}$. Therefore it will take $8-12 \mathrm{~min}$ utes to infuse enough fluid to resuscitate a patient who has lost 1 litre of blood. A smaller volume of an equally effective fluid is advantageous as it may be administered more rapidly and reduces the total amount of fluid required. Hypertonic saline may also help reduce the total fluid requirement for resuscitation. In a study (14) to see the effects of hypertonic saline followed by conventional fluid therapy, a porcine model of haemorrhagic shock was used. Bled pigs were given either $10 \mathrm{mls} / \mathrm{kg}$ hypertonic saline or $20 \mathrm{mls} / \mathrm{kg}$ lactated Ringer's solution as initial fluid for resuscitation, followed by a standard infusion of lactated Ringer's solution until they were haemodynamically stable. The hypertonic saline group as expected had, early on, a much improved haemodynamic status shown by a significant improvement in cardiac index, mean arterial pressure, fall in systemic vascular resistance and a rapid resumption of urine production. It was also important that the total fluid requirements in the hypertonic saline group were $60 \%$ less than in the conventional group. Various concentrations of saline in the hypertonic solutions have been used in these studies. Of all the different concentrations used, $7.5 \% \mathrm{NaCl}$ has been shown to be the most effective (13). Swine subjected to lethal haemorrhage were resuscitated with equal volumes of $0.9 \%, 5 \%$, $7.5 \%$ and $10 \%$ saline. There was a significant improvement in survival in the $7.5 \%$ group. Ten percent saline was shown to be toxic and rapidly fatal possibly due to a negative inotropic effect on the myocardium.

Hypertonic saline produces a rapid expansion of plasma volume by an acute rise in plasma osmolality of around
20 milliosmols. This generates a transcapillary force of up to $40 \mathrm{mmHg}$ drawing fluid into the vascular compartment, by an effect of the osmolality, not the sodium ion. Therefore, other hypertonic solutions were used (15) to see if they were any more effective than hypertonic saline. All solutions in the study rapidly returned the haemodynamic $\Rightarrow$ variables to normal levels. Solutions of mannitol and hypertonic glucose however produced a large diuresis and big fluid losses and hypertonic sodium bicarbonate caused severe alkalosis, so hypertonic saline was judged as the most effective.

\section{Hypertonic Saline Dextran Studies}

Colloid solutions have not been directly compared to hypertonic saline for resuscitation in hypovolaemic shock. Colloids are better retained in the vascular compartment than crystalloids and can replace blood on a volume for volume basis. They do, however, have side effects such as interfering with blood cross-matching and coagulation and can cause anaphylactic reactions. They are held at field ambulance level for resuscitation but are not widely used in pre-hospital resuscitation, the standard fluid used by American emergency medical technicians is Ringer's lactate. Colloid solutions however can be added to hypero tonic saline to produce an effective resuscitation fluis $T$ Studies have shown (16) that a solution of $7.5 \%$ saline $6 \%$ dextran 70 given in small volumes, usually $4 \mathrm{mls} / \mathrm{k}$ \% not only produces an immediate improvement in bloof pressure and cardiac output, but also sustains this response and improves survival.

Hypertonic saline was compared directly to hypertonic saline in dextran in pigs subjected to lethal haemorrhage (17). The animals were resuscitated with $4 \mathrm{mls} / \mathrm{kg}$ boluses of different solutions as the sole resuscitation fluids. Animals that received $7.5 \%$ saline in $6 \%$ dextran 70 had significantly improved survival after 4 hours compared to those receiving hypertonic saline alone, $6 \%$ dextran 70 in normal saline or normal saline.

The colloid does not need to be dextran 70, hypertonic saline combined with hetastarch (18) also produces a sustained cardiovascular response in the resuscitation of bled sheep. The response, however, when compared to dextran 70 and hypertonic saline, was slightly less effective probably due to the higher oncotic pressures generated by dextran. Hypertonic saline dextran solutions have also been $\frac{\mathscr{J}}{\vec{F}}$ evaluated for their role in pre-hospital resuscitation. In a $\dot{\sigma}$ study designed to mimic pre-hospital conditions the bene- 3 . fits of small volume resuscitation with hypertonic saline 8 dextran verses the risks of obligatory time delay to initiate the infusion were examined (19).This showed that hyper- o tonic saline dextran improved mean arterial pressure, cardiac index and oxygen consumption in the pre-hospital phase and attenuated the oxygen debt and secondary lactic acidosis in the wait and treat group despite the time delay. 0 The time spent in administration of the small volume of $N$ fluid was short and the total dose was quickly administered. These studies have led to the adoption of hypertonic 
solutions combined with dextran 70 as the main fluid used in studies concerned with small volume resuscitation.

\section{Human Trials with Hypertonic Saline Dextran Solution}

The first trial using hypertonic saline dextran in humans was in acutely injured adults with systolic blood pressures less than $100 \mathrm{mmHg}$ who were undergoing helicopter transfer to level 1 trauma centres (20). Twenty patients were given $250 \mathrm{mls}$ of either hypertonic saline dextran or Ringer's lactate initially, followed by conventional resuscitation. The group who received the hypertonic saline dextran showed improved, although not statistically significant, survival. Later this study was extended to 166 patients $(21)$ in a prospective randomised double-blind clinical trial. The patients received either $250 \mathrm{mls}$ of $7.5 \%$ sodium chloride in $6 \%$ dextran 70 or lactated Ringer's solution prior to normal resuscitation. The former group had better blood pressures on arrival in the emergency room, and their survival rates to discharge from hospital were improved but not significantly so.

Patients with severe head injuries, however, who were in the hypertonic saline dextran group did show a significant increase in survival to discharge from hospital.

Patients in this study had their serum electrolytes and osmolalities measured within 30 minutes of arriving at hospital. There were increases in the sodium, chloride and osmolalities in the hypertonic saline group, but these were not statistically significant and, more importantly, there were no adverse effects attributable to hypernatraemia or increased serum osmolality. Neither were there any complications due to dextran such as increased bleeding, interference with cross-matching blood or anaphylaxis. Further human trials evaluated the effect of hypertonic saline dextran given to patients in the emergency room. In a prospective randomised placebo controlled double-blind trial (22), $7.5 \%$ saline in $6 \%$ dextran was given to hypotensive hypovolaemic patients and, as demonstrated in other similar studies $(23,24)$, despite producing satisfactory resuscitation and having no adverse effects, there was no significant improvement in patient survival. The encouraging nature of these trials prompted the initiation of a multicentre trial in the USA to evaluate the safety and efficacy of $7.5 \% \mathrm{NaCl}$ in $6 \%$ dextran 70 in post traumatic hypotension (25). This study included 422 patients over a 13 month period. Survival was improved but they were not able to demonstrate statistical significance (it was estimated that 1200 patients would need to be studied to establish statistical validity). These studies conclude that the current status of hypertonic saline dextran is as a safe agent with definite beneficial effects when used in the resuscitation of hypovolaemic patients, but has not yet been shown to significantly alter patient survival.

\section{Mechanisms of Action of Hypertonic Solutions}

There are several mechanisms by which hypertonic saline solutions produce their effects. The acute rise in plasma osmolality causes an immediate expansion of the intravascular volume as fluid is drawn into the vascular compartment from the interstitial and intracellular spaces. The presence of the colloid augments the response by retaining this fluid intravascularly. The increase in cardiac output is attributable to an increase in venous return and cardiac filling augmenting stroke volume.

There is a vasodilatory action of hypertonic saline on pre capillary vessels causing a reduction in peripheral vascular resistance with a beneficial reduction in afterload and at the same time, constriction of the venous capacitance vessels. There is also a beneficial reduction in blood viscosity. Regional blood flow is improved and there is an improvement in urine output as a result of both improved renal perfusion and a decrease in renal vascular resistance.

Hypertonic saline has a direct effect on the myocardium increasing contractility, and dextran is thought to have an effect, scavenging free radicals released in hypoxic states that depress myocardial function.

Other mechanisms proposed for the mechanism of action of hypertonic saline are a vagally mediated response to the solution on pulmonary osmoreceptors (26). Sodium and chloride ions may also effect the cell membrane and help to restore the membrane potential damaged by the hypovolaemic insult. This will then pre-vent shifts of fluid into the cell as occurs in the later phases of haemorrhagic shock as cell metabolism is dis rupted.

The advantages of hypertonic saline dextran therefore are, a rapid and sustained improvement in the haemody namic status of the patient effective with small volumes i.e. $250 \mathrm{mls}$ or $4 \mathrm{mls} / \mathrm{kg}$. There may also be reduction in the total volume of fluid required for resuscitation $(14,23)$ but this finding is not consistent in all studies (25). Patient survival is improved with the use of hypertonic saline dextran when given early on in resuscitation. Although this increased survival has not been shown to be significant, patients with head injury (21) did have a statistically improved survival when resuscitated with hypertonic solutions. This is probably due to the fact that less resuscitation fluid was required in a group of patients who are at risk from cerebral oedema. There is also a beneficial effect of hypertonic saline dextran in reducing brain swelling, and by improving cardiac output early on, oxygen supply to the brain is preserved.

A similar protective effect against tissue oedema is also seen in the lung.

\section{Complications of Hypertonic Saline Dextran}

The potential disadvantages of hypertonic saline dextran are acute hypernatraemia and hyperosmolality causing irritability, muscle twitching, hyperreflexia, coma, seizures and death due to cellular dehydration. Demyelination and central pontine myelinolysis are also complications. A $7.5 \%$ solution of sodium chloride contains 1283 mmols/l of sodium and chloride ions, and has an osmolality of $2567 \mathrm{mosmol} / \mathrm{kg}$ compared to normal saline (0.9\%) 
which has a sodium and chloride content of $150 \mathrm{mmols} / 1$ and an osmolality the same as plasma (isotonic).

The total dose of sodium administered has been measured in studies $(13,14)$, and the amount of sodium received by the animals was equivalent or less in the ones given hypertonic saline compared to those receiving ordinary resuscitation. This is a reflection of the smaller volumes of fluid used in the former group.

Changes in electrolyte concentrations do occur and most studies $(17,21,22,23,25)$ report significant rises early on in serum sodium and osmolality in patients receiving hypertonic saline. Hypokalaemia also occurs but there were no adverse effects. The rapid expansion of the circulation, however, rapidly dilutes the electrolytes back to normal values and the rise in osmolality correlates more with serum alcohol levels than the use of hypertonic resuscitation fluids (22).

Anaphylatic reactions, cross-matching difficulties and coagulopathy are all potential problems with dextran, but again, probably due to the small doses involved, these complications have not been reported. The irritant nature of the solutions especially if they extravasate is a potential problem; again this has not been reported.

One of the major concerns with the use of hypertonic saline dextran is that the rapid normalisation in blood pressure, cardiac output and tissue blood flow will aggravate blood loss in situations where there is uncontrolled haemorrhage. In a study to examine this problem (27), a rat model was used for controlled and uncontrolled haemorrhage. The two groups received either hypertonic saline or normal saline for resuscitation. The controlled haemorhage group had a significantly better cardiovascular response with hypertonic saline resuscitation than with normal saline whereas in the uncontrolled haemorrhage group, hypertonic saline, produced a rapid increase in bleeding, a precipitous fall in blood pressure and rapid death.

This work however, was challenged by a study using computer generated mathematical modelling to predict blood volume expansion with resuscitation fluid regimes of varied osmotic composition (28). With uncontrolled haemorrhage hypertonic saline dextran initially increased bleeding, but plasma volume expansion exceeded blood loss and the response to hypertonic saline dextran followed by Ringer's lactate was better than with Ringer's lactate alone.

\section{Conclusions}

The advantages of hypertonic saline dextran solutions can be summarised as being able to provide effective resuscitation in much smaller volumes, and therefore more quickly than conventional fluids. They lack serious side effects and help to reduce the total fluid requirements for resuscitation. They are potentially most valuable in the pre-hospital phase when transfer time to hospital is relatively short.
In a battlefield situation prolonged evacuation of casualties may negate these useful effects. There is also poten $\frac{\bar{D}}{2}$ tial for serious consequences if hypertonic solutions are given in large volumes by unskilled resuscitators who do not understand their use. There is, however, a need for? change in the whole philosophy of casualty treatment. The most likely causes of death in battle casualties are exsan-guination and head injuries (29) and the most importan factor improving survival of casualties is rapid evacua $\overline{\bar{n}}$. tion. The six to eight hour delays seen in the second world war were reduced to two to four hours in the Korean wan and Vietnam by using dedicated helicopters for casualtyes evacuation, and the survival rate for casualties was greatlyo improved.

Modern conflicts do not produce vast numbers of casu alties but rather smaller numbers, albeit in isolated areas These smaller numbers of casualties should allow fo: more intensive resuscitation and with the use of heli-? copters, evacuation times can be much reduced. It is in this scenario that small volume resuscitation with hyper 0 tonic saline dextran solution could be most effectively. used. The solution given appropriately by trained medica personnel would provide rapid and sustained improves ments in haemodynamic status and would reduce the neg for large volumes of fluids for resuscitation. The ot potential advantage of hypertonic fluids is the improw outcome of patients with head injuries which account top a large number of battle casualties (29). Hypertonic sali dextran solution is, therefore, a pharmacological aggn that could be added to the armamentarium of military de tors concerned with forward resuscitation in specific narios. A certain level of medical expertise is required use hypertonic saline solutions effectively but in the context of helicopter evacuation and aggressive resuscitationő of smaller numbers of potentially more serious injuree $\mathbb{R}$ casualties, it certainly has a role to play.

\section{REFERENCES:}

1. J G Williams, T R D RILEy, R A Moody. Resuscita? tion experience in the Falkland Islands campaign. $B r$ Med $J$ 1983; 286: 775-777.

2. Kirby N G, Blackburn G. Field Surgery Pocke Book. London: HMSO 1981.

3. TRUNKEY D P. Is ALS necessary for pre-hospital care J Trauma 1984; 24: 86-87.

4. Smith J P, Bodai B I, Hill A S, Frey C F. Pre-hospi tal stabilisation of critically injured patients: a failedi concept. J Trauma 1985; 25: 65-70.

5. KAWESKi S M, Sise M J,Virgilio R W. The effects? of prehospital fluids on survival in trauma patients. $J_{\supset}$ Trauma 1990; 30: 1215-1219.

6. HOWORTH P J N. The role of isotonic and hypertonion solutions in the resuscitation of shocked patients. $J R^{\circ}$ Army Med Corps 1985; 131: 100-104.

7. SILBERT S. The treatment of thromboangiitis obliter-w ans by intravenous injection of hypertonic salt solu 
tion: A preliminary report. JAMA $1926 ; 86: 1759$ 1761.

8. Brooks D K, Williams W G, Manley R W. Osmolar and electrolyte change in haemorrhagic shock. Lancet 1963; 1: 521-527.

9. Baue a E, Tragus E T, Parkins W M. A comparison of isotonic and hypertonic solutions and blood on flow and oxygen consumption in the initial treatment of haemorrhagic shock. $J$ Trauma 1967; 7: 743-756.

10. Velasco I T, Pontieri V. Hyperosmotic NaCI and severe haemorrhagic shock. Am J Physiol 1980; 239: 4664-4673.

11. Nakayama S, Sibley L, Gunther R A, Holcroft J W, Kramer G C. Small volume resuscitation with hypertonic saline $(2400 \mathrm{mOsmol} / 1)$ during haemorrhagic shock. Circ Shock 1984; 13: 149-159.

12. Bitterman H, Triolo J, Lefer A M. Use of hypertonic saline in the treatment of haemorrhagic shock. Circ Shock 1987; 21: 27.1-283.

13. Traverso I W, Bellamy R F, Hollenbach S J, WILCHER L D. Hypertonic sodium chloride solutions: effects on haemorrhage in swine. J Trauma 1987; 27: 32-39.

14. Stanford G G, Patterson C R, Payne L, Fabian T C. Hypertonic saline resuscitation in a porcine model of severe haemorrhagic shock. Arch Surg 1989; 124: 733-6

15. Smith G J, Kramer G C, Perron P, Nakayama $S$, GUNTHER R A, Holcroft J W. A comparision of several hypertonic solutions for resuscitation of bled sheep. J Surg Res 1985; 39: 517-528.

16. Maningas P A, DeGuzman L R, Tillman F J, et al. Small volume infusion of $7.5 \% \mathrm{NaCl}$ in $6 \%$ Dextran 70 for the treatment of severe haemorrhagic shock in swine. Ann Emerg Med 1986; 15: 1131-1137.

17. Wade C E, Hannon J P, Bossone C A, et al. Resuscitation of concious pigs following haemorrhage: comparative efficacy of small-volume resuscitation. Circ Shock 1989; 29: 193-204.

18. Kramer G C, Walsh J C, Perron P R, Gunther R A, Holcroft J W. Comparison of hypertonic saline/dextran versus hypertonic saline/hetastarch for resuscitation of hypovolaemia. Braz J Med Biol Res 1989; 22: 279-82.

19. Siritongtaworn P, Moore E E, Marx J A, et al. The benefits of $7.5 \% \mathrm{NaC} 1 / 6 \%$ dextran 70 (HSD) for prehospital resuscitation haemorrhagic shock: improved oxygen transport. Braz J Med Biol Res 1989; 22: 275-8.

20. Holcroft J W, Vasser M J, Turner Derlet R W, KRAMER G C. $3 \% \mathrm{NaC1}$ and 7.5\% NaC1/Dextran in resuscitation of severely injured patients. Ann Surg 1987; 206:279-288.

21. Vassar M J, Perry C A, Gannaway W L, HolCROFT J W. $7.5 \%$ sodium chloride/dextran for resuscitation of trauma patients undergoing helicopter transport. Arch Surg 1991; 126: 1065-72.

22. Holcroft J W, Vassar M J, Perry C a, Gannaway W L, Kramer G C. Use of a $7.5 \% \mathrm{NaCl} / 6 \%$ Dextran 70 solution in the resuscitation of injured patients in the emergency room. Prog Clin Biol Res 1989; 299: 331-8.

23. Younes R N, Aun F, Accioly C Q, et al. Hypertonic solutions in the treatment of hypovolaemic shock: A prospective, randomised study in patients admitted to the emergency room. Surgery 1992; 111: 380-5.

24. Holcroft J W, Vassar M J, Perry C a, Gannaway W L, Krammer G C. Perspectives on clinical trials for hypertonic saline/dextran solutions for the treatment of traumatic shock. Braz J Med Biol Res 1989; 22: 291-3.

25. Mattox K L, Maningas P A, Moore E E et al. Prehospital hypertonic saline/dextran infusion for posttraumatic hypotension. The USA multicentre trial. Ann Sur 1991; 213: 482-91.

26. Lopes O U, Pontierei V, Rocha E, Silva M J N, Velasco I T. Hyperosmotic $\mathrm{NaCl}$ and severe haemorrhagic shock: Role of the innervated lung. $A m J$ Physiol 1981; 241: H883-H890

27. Gross D, Landau E, Assalia A, Krausz M M. Is hypertonic saline resuscitation safe in "uncontrolled" haemorrhagic shock? J Trauma 1988; 28: 751-756.

28. Mazzoni M, P, Borgstrom P, Arfors K E, IntagliETTAM. The efficacy of iso- and hyperosmotic fluids as volume expanders in fixed volume and uncontrolled haemorrhage. Ann Emerg Med 1990; 9: 350-8.

29. Bellamy R F. The causes of death in conventional land warfare: Implications for combat casualty care research. Milit Med 1984; 149: 55-62. 